\title{
A Multisampling Reporter System for Monitoring MicroRNA Activity in the Same Population of Cells
}

\author{
Pei-Chen Huang, ${ }^{1}$ Chih-Ying Chen, ${ }^{1,2}$ Feng-Yuan Yang, ${ }^{2}$ and Lo-Chun Au ${ }^{1,2}$ \\ ${ }^{1}$ Institute of Biotechnology in Medicine, Department of Biotechnology and Laboratory Science in Medicine, \\ National Yang-Ming University, Taipei 11221, Taiwan \\ ${ }^{2}$ Department of Medical Research and Education, Taipei Veterans General Hospital, Taipei 11217, Taiwan
}

Correspondence should be addressed to Lo-Chun Au, lcau@vghtpe.gov.tw

Received 22 July 2009; Revised 21 October 2009; Accepted 5 November 2009

Recommended by Paul Higgins

MicroRNAs (miRNAs) downregulate gene expression by binding to the partially complementary sites in the $3^{\prime}$ untranslated region (UTR) of target mRNAs. Several methods, such as Northern blot analysis, quantitative real-time RT-PCR, microarray, and the luciferase reporter system, are commonly used to quantify the relative level or activity of miRNAs. The disadvantage of these methods is the requirement for cell lysis, which means that several sets of wells/dishes of cells must be prepared to monitor changes in miRNA activity in time-course studies. In this study, we developed a multisampling reporter system in which two secretable bioluminescence-generating enzymes are employed, one as a reporter and the other as an internal control. The reporters consist of a pair of vectors containing the Metridia luciferase gene, one with and one without a duplicated miRNA targeting sequence at their $3^{\prime} \mathrm{UTR}$, while the other vector coding for the secreted alkaline phosphatase gene is used as an internal control. This method allows miRNA activity to be monitored within the same population of cells over time by withdrawing aliquots of the culture medium. The practicability and benefits of this system are addressed in this report.

Copyright (C) 2009 Pei-Chen Huang et al. This is an open access article distributed under the Creative Commons Attribution License, which permits unrestricted use, distribution, and reproduction in any medium, provided the original work is properly cited.

\section{Introduction}

MicroRNAs (miRNAs) are small noncoding RNAs found in plants and animals which downregulate gene expression by binding to complementary sites on the $3^{\prime}$ untranslated region (UTR) of target mRNAs. As a result, the targeted mRNAs are degraded if the miRNA and target sequence are a perfect match or their translation is inhibited [1]. Over 800 different miRNAs are found in the human genome (miRBase: http://microrna.sanger.ac.uk/; Sources of predicted miRNA target: http://microrna.org), and each miRNA may have hundreds of target mRNAs. For this reason, miRNAs have profound effects on global gene expression and are known to play roles in various cellular processes, such as development, differentiation, proliferation, and apoptosis [2-4]. A number of miRNAs have been linked to human cancer $[1,5]$.

Several methods are used to detect the relative level or activity of miRNAs, including Northern blot analysis [6], quantitative real-time RT-PCR [7], microarray [6, 8], and the firefly luciferase reporter system $[7,9]$. To circumvent the tediousness and accuracy-compromising drawbacks of the lysis of the test cells that is required in all the above methods, in this study, we developed a multisampling reporter system that makes it possible to monitor changes in miRNA activity over time within the same population of cells by sampling the culture medium, thus avoiding the need for a lysis step. Briefly, the system contains two distinct vectors that encode different secretable bioluminescence-generating enzymes. The reporters consist of a pair of vectors containing the Metridia luciferase (MLuc) gene with or without a duplicated miRNA targeting sequence at their $3^{\prime}$ UTR, while the other vector coding for the secreted alkaline phosphatase (SEAP) gene is used as an internal control. The expression of all three vectors is driven by the same SV40 promoter. MLuc, a naturally secreted luciferase derived from Metridia longa [10], oxidizes coelenterazine and produces luminescence. Although placental alkaline phosphatase is a membranebound protein, SEAP is genetically engineered and lacks the membrane-anchoring domain [11]. The heat-resistant SEAP hydrolyzes the substrate 1, 2-dioxetane and generates 
luminescence [12]. Taking advantage of these features, we can sample small aliquots of medium over time and keep the monitored cells intact. In this study, this system was used to monitor the activity of miR-15/miR-16, which may downregulate the expression of BCL-2, an antiapoptotic protein $[13,14]$, by binding to their target sequence in the $3^{\prime}$ UTR of the $B c l-2$ gene $[15,16]$.

\section{Materials and Methods}

2.1. Plasmid Construction. The MLuc gene from the pMetLuc-Control plasmid (Clotech Inc., Palo Alto, CA, USA) was amplified by PCR using the forward primer $5^{\prime}$-CGAAGCTTATGGACATCAAGGTGGTG- ${ }^{\prime}$ and the reverse primer $5^{\prime}$-TATGATCTAGAGTCGCGG-3' (HindIII and XbaI restriction sites are indicated in bold letters). The amplicon was cut by HindIII and XbaI. pGL3Control (Promega Inc., Madison, WI, USA) was cut using the same restriction enzymes to remove the firefly luciferase gene, and the MLuc amplicon was inserted. The recombinant vector was designated pMLuc. Two complementary oligonucleotides 5'TCTAGAAATATCCAA TCCTGTGCTGCTATCCTGCCAACAATATCCAATCCTGT GCTGCTATCTAGA3' and 5'AGATCTTTATAGGTTAGG ACACGACGATA G G A C G G T T GTTATAGGTTAGGACA CGACGATAGATC3' containing two miR15/miR-16 target sequences (the $3^{\prime}$ UTR target site in human Bcl-2 mRNA), indicated in bold letters, were annealed. The doublestranded oligonucleotide was inserted into the XbaI site of pMLuc which is located downstream of the stop codon of the $M L u c$ gene. The recombinant vector obtained was named pMLuc-UTR. pSEAP2-Control (Clotech Inc.) containing the SEAP gene was used as an internal control.

2.2. Pre-miRNA. Pre-miR-16 and the negative control premiR (NC miR) were purchased from Ambion (Austin, TX, USA).

2.3. Cell Culture and Transfection. HEK293T and HepG2 cells (purchased from the Bioresource Collection and Research Center, Taiwan) were cultured in DMEM containing $10 \%$ fetal bovine serum (both from Gibco BRL, Gaithersburg, $\mathrm{MD}, \mathrm{USA}$ ) at $37^{\circ} \mathrm{C}$ in a humidified $\mathrm{CO}_{2}$ incubator. For transfection, the cells were grown in 6-well plates to $70 \%$ confluence. Transfection with plasmids and pre-miRNA was performed using a previously described protocol [17].

2.4. Assays of MLuc and SEAP Activity. Culture medium was collected and centrifuged, then $20 \mu \mathrm{L}$ aliquots of the cellfree medium diluted in $180 \mu \mathrm{L}$ of $0.9 \% \mathrm{NaCl}$ were assayed for MLuc and SEAP activity using kits purchased from Clotech. For the MLuc assay, the $10 \times$ MLuc substrate stock was diluted to $1 \times$ in reaction buffer then diluted to $0.5 \times$ in double-distilled $\mathrm{H}_{2} \mathrm{O}$; then $5 \mu \mathrm{L}$ of the substrate/buffer solution and $50 \mu \mathrm{L}$ of the diluted sample were mixed and loaded in 96-well black plates to detect luminescence. For the SEAP assay, $30 \mu \mathrm{L}$ of $1 \times$ dilution buffer and $10 \mu \mathrm{L}$ of the diluted sample were mixed and incubated at $65^{\circ} \mathrm{C}$ for 30 minutes and $4^{\circ} \mathrm{C}$ for 2 minutes in 96-well black plates, then $40 \mu \mathrm{L}$ of SEAP substrate solution was added and the samples incubated at room temperature for 30 minutes before detecting the luminescence.

2.5. Quantification of miR-16 by qRT-PCR. HepG2 cells were grown in $6 \mathrm{~cm}$ Petri dishes to $70 \%$ confluence then were treated for different periods with $100 \mu \mathrm{M}$ epigallocatechin gallate (EGCG). Total RNA was extracted from the cells using Trizol reagent (Invitrogen). qRT-PCR analysis for miRNAs was performed using TaqMan MicroRNA assays kit (Applied Biosystem, Foster City, CA, USA). Fifty nanograms of total RNA were subjected to reverse transcription (RT) using miR16 and U6 RT primers (has-miR-16 UAGCCAGCACGUAAAUAUUGCG; U6 CGCAAGGAUGACACAC GCAAAUUCGUG AAGCGUUCCAUAUUUUU) purchased from Applied Biosystem. RT was performed on a PCR machine $\left(16^{\circ} \mathrm{C}\right.$ for 30 minutes, $42^{\circ} \mathrm{C}$ for 30 minutes, and $85^{\circ} \mathrm{C}$ for 5 minutes). Real-time PCR was performed using an ABI PRISM 7900 (Applied Biosystem) and specific primer sets for miR-16 and U6 RNAs from Applied Biosystem. The conditions for the real-time PCR were $95^{\circ} \mathrm{C}$ for 10 minutes and 40 cycles of $95^{\circ} \mathrm{C}$ for 30 seconds and $60^{\circ} \mathrm{C}$ for 1 minute. The $\Delta C t$ was calculated by subtracting the $C t$ for U6 small nuclear RNA from that for miR-16. The $\Delta \Delta C t$ was calculated by subtracting the $\Delta C t$ for the untreated control from that for the EGCG-treated sample. The fold change in miR-16 levels was calculated as $\log 2^{-\Delta \Delta C t}$.

\section{Results and Discussion}

To monitor the activity of miRNA in vivo without destroying the test cells, we devised a secretable reporter system consisting of three vectors that allows accurate and continuous measurement of the test miRNA. All 3 vectors (pMLuc, pMLuc-UTR, and pSEAP2-Control) were designed to use the same SV40 promoter to regulate the expression of the reporters or the internal control. pSEAP2-Control serves the dual role of (1) normalizing the transfection efficiency and (2) normalizing the activity of the SV40 promoter, which may differ in the context of various treatments. We first examined the signal-to-noise (background) ratio of the system and the stability of the two enzymes in culture medium to freeze thawing to determine whether samples from a time-course study could be frozen then assayed simultaneously. To examine these parameters, HEK293T cells were transfected with pMLuc or pSEAP2-Control, then $1 \mathrm{~mL}$ of culture medium was withdrawn 24 hours after transfection and centrifuged. The cell-free medium was then incubated at $37^{\circ} \mathrm{C}$ in a $\mathrm{CO}_{2}$ incubator and $20 \mu \mathrm{L}$ aliquots were withdrawn at different time points and assayed for MLuc or SEAP activity. The results, shown in Figure 1(a), indicate that the background signals from DMEM and conditioned medium from nontransfected cells were $<0.1 \%$ of that from conditioned medium from pMLuc- or pSEAP2Control-transfected cells. The secreted enzymes were stable 

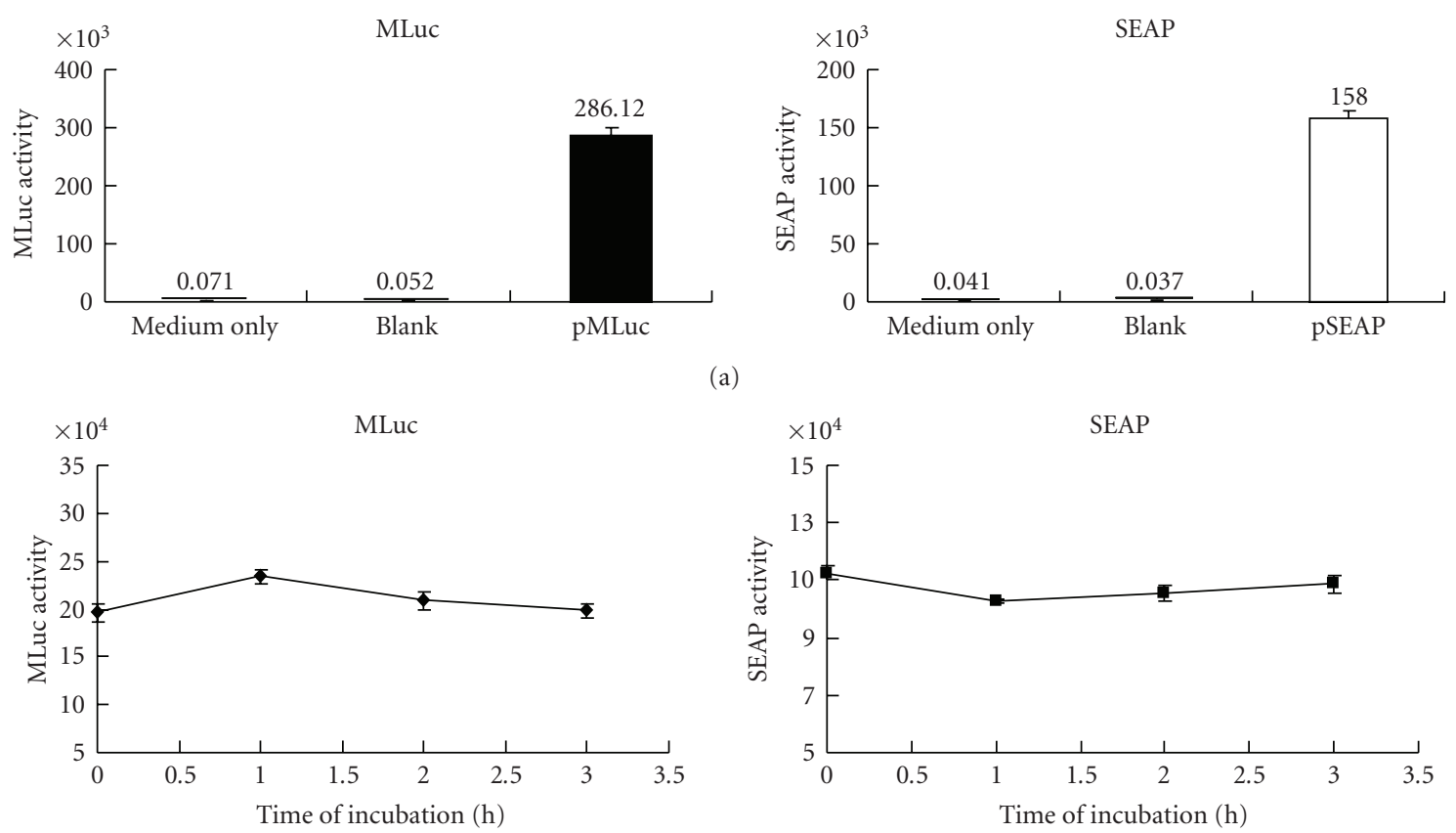

(a)

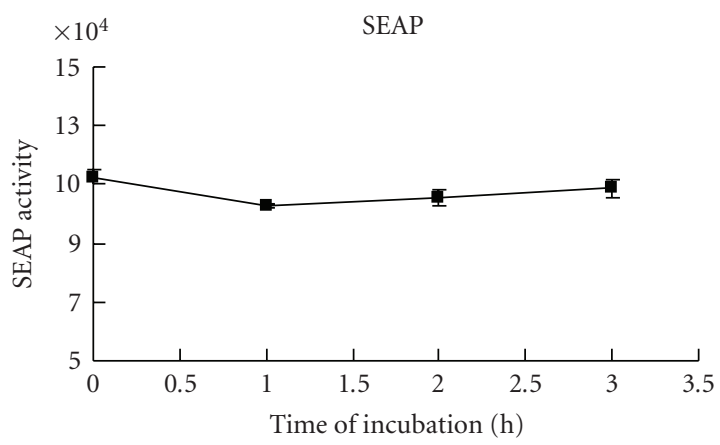

(b)
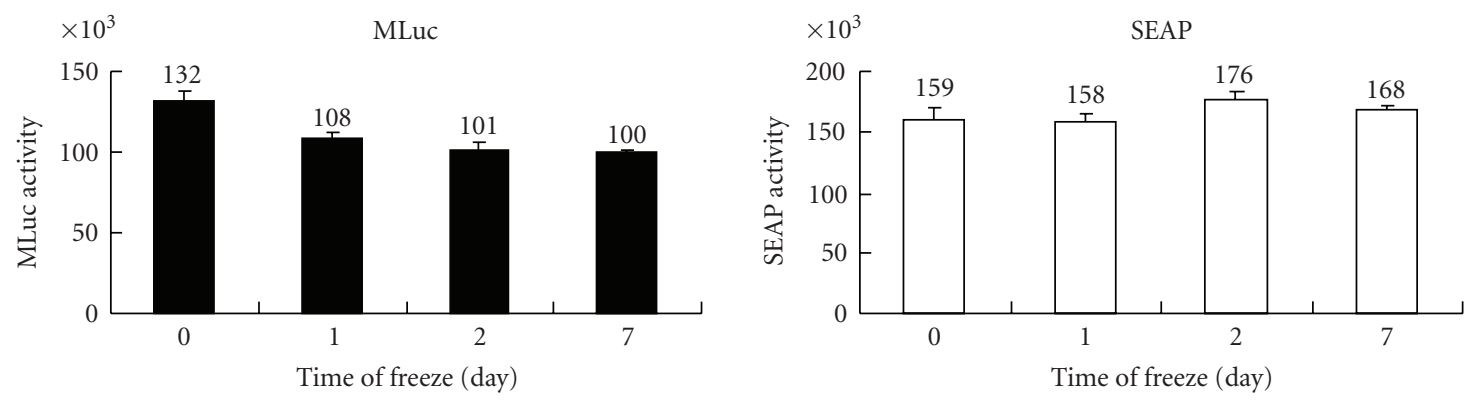

(c)

FIGURE 1: Characterization of the multisampling reporter system. HEK293T cells were grown in 6-well plates to $70 \%$ confluency, then some were left untreated and others were transfected with $0.5 \mu \mathrm{g}$ of pMLuc or pSEAP2-Control for 24 hours. (a) An aliquot of the medium was assayed for MLuc (left) or SEAP (right). (b) The culture medium was collected and incubated at $37^{\circ} \mathrm{C}$ in a $5 \% \mathrm{CO}_{2}$ humidified chamber and aliquots were withdrawn at different time points and assayed for MLuc (left) or SEAP (right). (c) Medium samples were frozen at $-20^{\circ} \mathrm{C}$ in separate Eppendorf tubes, and thawed at the indicated times, and assayed. The data presented are the mean \pm SD of triplicates.

in culture medium at conditions of $37^{\circ} \mathrm{C}$ and $5 \% \mathrm{CO}_{2}$ for several hours (Figure 1(b)). A single freezethaw cycle reduced MLuc activity by $20 \%$, but activity was not further reduced by freezing for 7 days (Figure 1(c)). Freeze-thaw and freezing for 7 days had no effect on SEAP activity (Figure 1(c)). This shows that we can collect and freeze all the samples from a single experiment and test them later without compromising the accuracy of the results. Thus, MLuc and SEAP are practicable for a multisampling reporter assay.

To test whether our system faithfully reflected the cellular activity of miR-15/miR-16, HEK293T cells were cotransfected with pSEAP2-Control, pMLuc or pMLucUTR, and pre-miR-16 or NC miR. The culture medium was replaced with fresh medium at 16, 24, and 36 hours after transfection and aliquots of medium were taken 2 hours after each medium change and assayed for MLuc and SEAP. The MLuc/SEAP activity ratios after various treatments are shown in Figure 2(a). The results showed that the ratios for the pMLuc-UTR + pre-miR-16 group (black bars) were 2to 3 -fold lower than those for the pMLuc-UTR + NC miR group (gray bars), indicating that pre-miR-16 suppressed the expression of Mluc. In addition, the ratios for the pMLucUTR + NC miR group (gray bars) were 1.5-fold lower than those for the pMLuc + NC miR group (white bars). This is because endogenous miR-15/miR-16 can target the MLucUTR mRNA generated from pMLuc-UTR. Cotransfection with pMLuc, which does not contain miR-15/miR-16 target sites, resulted in a higher ratio. These results indicate that our reporting system can be used to detect miR-15/miR16 activity without disrupting the cells. The fourth group pMLuc + pre-miR-16 (hatched bars) showed higher ratios than those of the pMLuc + NC miR group (white bars), but a similar trend. This may be due to some global effect of miR-16 on the treated cells. In addition, by monitoring 


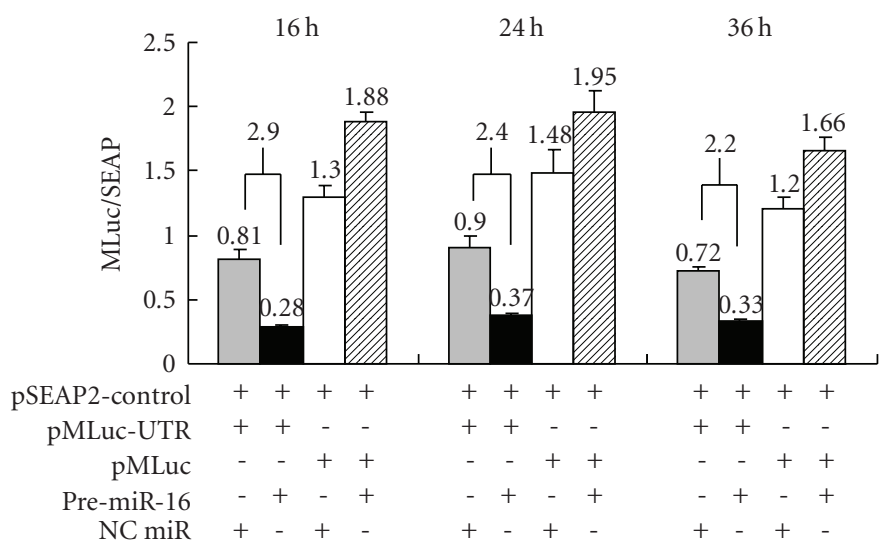

(a)

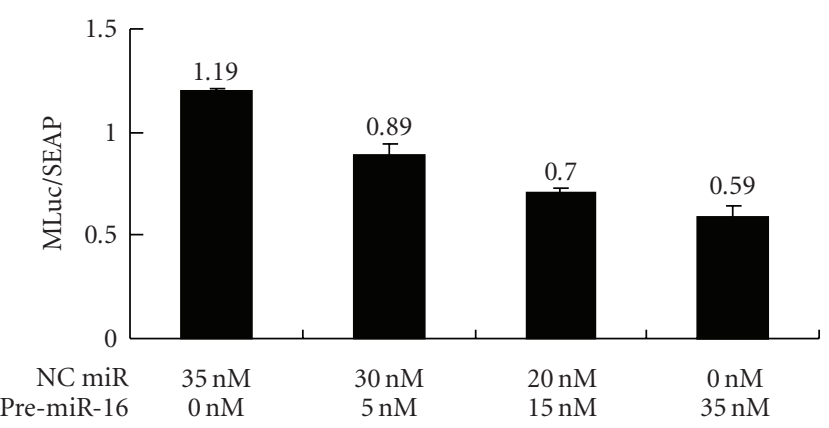

(b)

FIGURE 2: Reporting ability of the multisampling reporter system. HEK293T cells were grown in 6-well plates to 70\% confluency. (a) The cells were cotransfected with $0.5 \mu \mathrm{g}$ of pSEAP2-Control, $0.5 \mu \mathrm{g}$ of pMLuc or pMLuc-UTR, and $30 \mathrm{nM}$ pre-miR-16 or NC miR. The medium was replaced with fresh medium at different time points after transfection, and an aliquot of medium was withdrawn 2 hours later and assayed for MLuc and SEAP. (b) The cells were cotransfected with $0.5 \mu \mathrm{g}$ of pMLuc-UTR and pSEAP2-Control, together with different concentration of pre-miR-16 (0-35 nM) and NC miR (35-0 nM), then, 24 hours later, an aliquot of medium was withdrawn from each culture medium and assayed. The data presented are the mean $\pm \mathrm{SD}$ of triplicates.

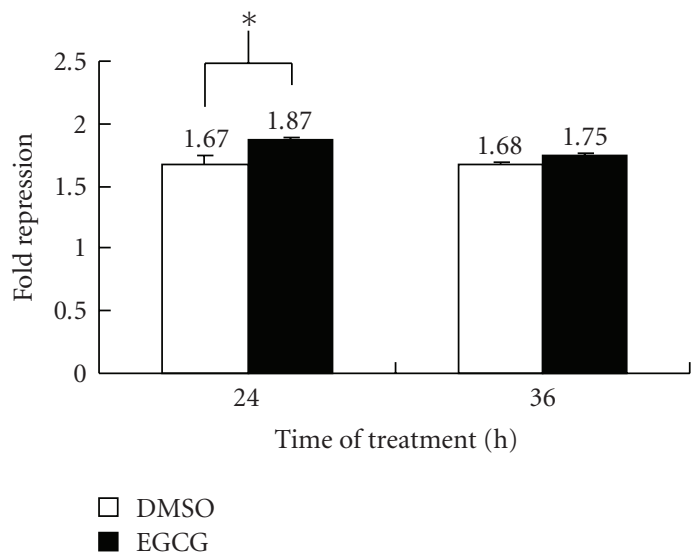

(a)

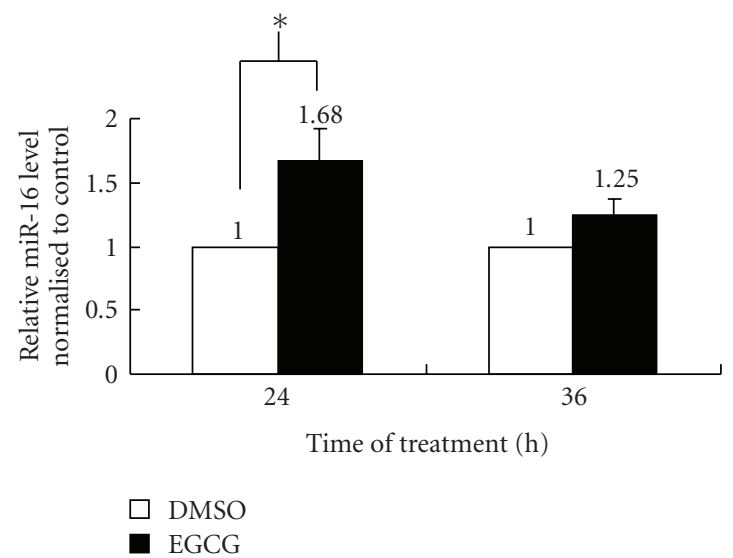

(b)

FIGURE 3: Increase in miR-16 activity after EGCG treatment of HepG2 cells. HepG2 cells were grown in $5 \mathrm{~mL}$ of DMEM in $6 \mathrm{~cm}$ Petri dishes to $70 \%$ confluency. (a) The cells were cotransfected for 6 hours with $4 \mu \mathrm{g}$ of pMLuc or pMLuc-UTR and $4 \mu \mathrm{g}$ of pSEAP2-Control, then $5 \mu \mathrm{L}$ of $100 \mathrm{mM}$ of EGCG (dissolved in DMSO) or DMSO was added. The medium was replaced with fresh medium at 24 and 36 hours and an aliquot of medium was withdrawn 2 hours later and assayed for MLuc and SEAP. The fold repression is defined as the MLuc (from pMLuc)/SEAP ratio divided by the MLuc (from pMLuc-UTR)/SEAP ratio. (b) The cells were treated with or without EGCG for 24 or 36 hours, then total RNA was extracted and subjected to qRT-PCR to detect levels of miR-16 and U6 small nuclear RNA (see Section 2 for details). The data presented are the mean \pm SD of triplicates; ${ }^{*} P<.05$.

the same population of cells at 16,24 , and 36 hours, we found that the difference between the pMLuc-UTR + NC miR group ratios (gray bars) and the pMLuc-UTR + premiR-16 group ratios (black bars) decreased from 2.9-fold to 2.4-fold, then to 2.2-fold. This suggests that there is a gradual reduction in miR-16 levels in cells with time. To test the sensitivity of our reporting system, HEK293T cells were cotransfected with pMLuc-UTR + pSEAP2-Control, and different concentrations of pre-miR-16 and NC miR (total concentration of the two $35 \mathrm{nM}$ ) and aliquots of medium were taken 24 hours after transfection and assayed for MLuc and SEAP activity. As shown in Figure 2(b), dose-dependent suppression of MLuc was seen in response to an increasing amount of pre-miR-16.

HepG2, a liver cancer cell line, is known to exhibit upregulation of miR-16 levels (1.5-fold) following treatment with epigallocatechin gallate (EGCG) [18]. We, therefore, used our reporter system to monitor changes in this endogenous miRNA. HepG2 cells were cotransfected with pSEAP2-Control and either pMLuc-UTR or pMLuc then were cultured in the presence or absence of EGCG. The result is shown in Figure 3, in which "fold repression" is defined as 
the MLuc (from pMLuc)/SEAP ratio divided by the MLuc (from pMLuc-UTR)/SEAP ratio. A significant increase in fold repression was seen in 24 hours of EGCG treatment compared to the blank control. A concomitant increase in miR-16 levels was confirmed by quantitative real-time RTPCR (Figure 3(b)). The role of miRNA in fine tuning of protein synthesis can be elicited from the results of Figures 2 (b) and 3. We have used our system to screen compounds which might up- or down-regulate a given miRNA. However, we found that some compounds had a direct effect on the enzymatic activities of MLuc and/or SEAP, leading to changes in the MLuc/SEAP ratio, while miRNA activities remained unchanged. We overcame this potential fault by calculating the fold repression instead of the MLuc/SEAP ratio, as the MLuc and SEAP activities were normalized in the calculation of the fold repression. We, therefore, strongly favor using the fold repression data for drug screening.

\section{Conclusions}

Our pMluc/pSEAP2-Control-based reporter facilitates multisampling for monitoring the activity of a given miRNA using culture medium. The extracellular reporters are less affected by intracellular modifications. Time-course studies can be performed using the same population of cells. Samples collected at constant interval after each medium refreshing provide an activity readout of a given miRNA in real time. Moreover, the same population of cells used in detecting miRNA activity can be used for RNA extraction for further determination of miRNA levels. This method offers the benefits of accuracy, convenience, and cost effectiveness. With no need for cell lysis or addition of protease inhibitors, our system is more applicable to robust high-throughput drug screening. Finally, the system is versatile, as it can be easily switched to monitor the activity of any given miRNA by inserting different synthesized miRNA-targeting sequences into the XbaI site of pMluc.

\section{Acknowledgments}

This study was supported by VGH grant V99C1-104 and grant of NSC 97-2320-B075-004-MY3 from the National Science Council, Taiwan. The authors also thank Wei-Chun Au for critical reading of the manuscript.

\section{References}

[1] A. Esquela-Kerscher and F. J. Slack, "Oncomirs-microRNAs with a role in cancer," Nature Reviews Cancer, vol. 6, no. 4, pp. 259-269, 2006.

[2] W. P. Kloosterman and R. H. Plasterk, "The diverse functions of microRNAs in animal development and disease," Developmental Cell, vol. 11, no. 4, pp. 441-450, 2006.

[3] B. D. Harfe, "MicroRNAs in vertebrate development," Current Opinion in Genetics and Development, vol. 15, no. 4, pp. 410$415,2005$.

[4] H.-W. Hwang and J. T. Mendell, "MicroRNAs in cell proliferation, cell death, and tumorigenesis," British Journal of Cancer, vol. 94, no. 6, pp. 776-780, 2006.
[5] G. A. Calin and C. M. Croce, "MicroRNA signatures in human cancers," Nature Reviews Cancer, vol. 6, no. 11, pp. 857-866, 2006.

[6] K. A. Cissell and S. K. Deo, "Trends in microRNA detection," Analytical and Bioanalytical Chemistry, vol. 394, no. 4, pp. 1109-1116, 2009.

[7] J. Y. Lee, S. Kim, W. H. Do, et al., "Development of a dual-luciferase reporter system for in vivo visualization of microRNA biogenesis and posttranscriptional regulation," Journal of Nuclear Medicine, vol. 49, no. 2, pp. 285-294, 2008.

[8] E. A. Miska, E. Alvarez-Saavedra, M. Townsend, et al., "Microarray analysis of microRNA expression in the developing mammalian brain," Genome Biology, vol. 5, no. 9, article R68, 2004.

[9] Y. Yang, R. Chaerkady, M. A. Beer, J. T. Mendell, and A. Pandey, "Identification of miR-21 targets in breast cancer cells using a quantitative proteomic approach," Proteomics, vol. 9, no. 5, pp. 1374-1384, 2009.

[10] S. V. Markova, S. Golz, L. A. Frank, B. Kalthof, and E. S. Vysotski, "Cloning and expression of cDNA for a luciferase from the marine copepod Metridia longa: a novel secreted bioluminescent reporter enzyme," Journal of Biological Chemistry, vol. 279, no. 5, pp. 3212-3217, 2004.

[11] J. Berger, J. Hauber, R. Hauber, R. Gieger, and B. R. Cullen, "Secreted placental alkaline phosphatase: a powerful new quantitative indicator of gene expression in eukaryotic cells," Gene, vol. 66, no. 1, pp. 1-10, 1988.

[12] I. Bronstein, J. J. Fortin, J. C. Voyta, et al., "Chemiluminescent reporter gene assays: sensitive detection of the GUS and SEAP gene products," Biotechniques, vol. 17, no. 1, pp. 172-177, 1994.

[13] R. J. Bold, S. Virudachalam, and D. J. McConkey, "Bcl2 expression correlates with metastatic potential in pancreatic cancer cell lines," Cancer, vol. 92, no. 5, pp. 1122-1129, 2001.

[14] A. Frenzel, F. Grespi, W. Chmelewskij, and A. Villunger, "Bcl2 family proteins in carcinogenesis and the treatment of cancer," Apoptosis, vol. 14, no. 4, pp. 584-596, 2009.

[15] A. Cimmino, G. A. Calin, M. Fabbri, et al., "miR-15 and miR-16 induce apoptosis by targeting Bcl2," Proceedings of the National Academy of Sciences of the United States of America, vol. 102, no. 39, pp. 13944-13949, 2005.

[16] L. Xia, D. Zhang, R. Du, et al., "miR-15b and miR-16 modulate multidrug resistance by targeting $\mathrm{Bcl} 2$ in human gastric cancer cells," International Journal of Cancer, vol. 123, no. 2, pp. 372379, 2008.

[17] Y.-F. Lai, Y.-J. Tseng, F.-Y. Yang, and L.-C. Au, "Mammalian cis-reporting plasmid may alter activities due to the derivation of host Escherichia coli strains," Analytical Biochemistry, vol. 376, no. 1, pp. 103-107, 2008.

[18] W. P. Tsang and T. T. Kwok, "Epigallocatechin gallate upregulation ofmiR-16 and induction of apoptosis in human cancer cells," Journal of Nutritional Biochemistry. In press. 

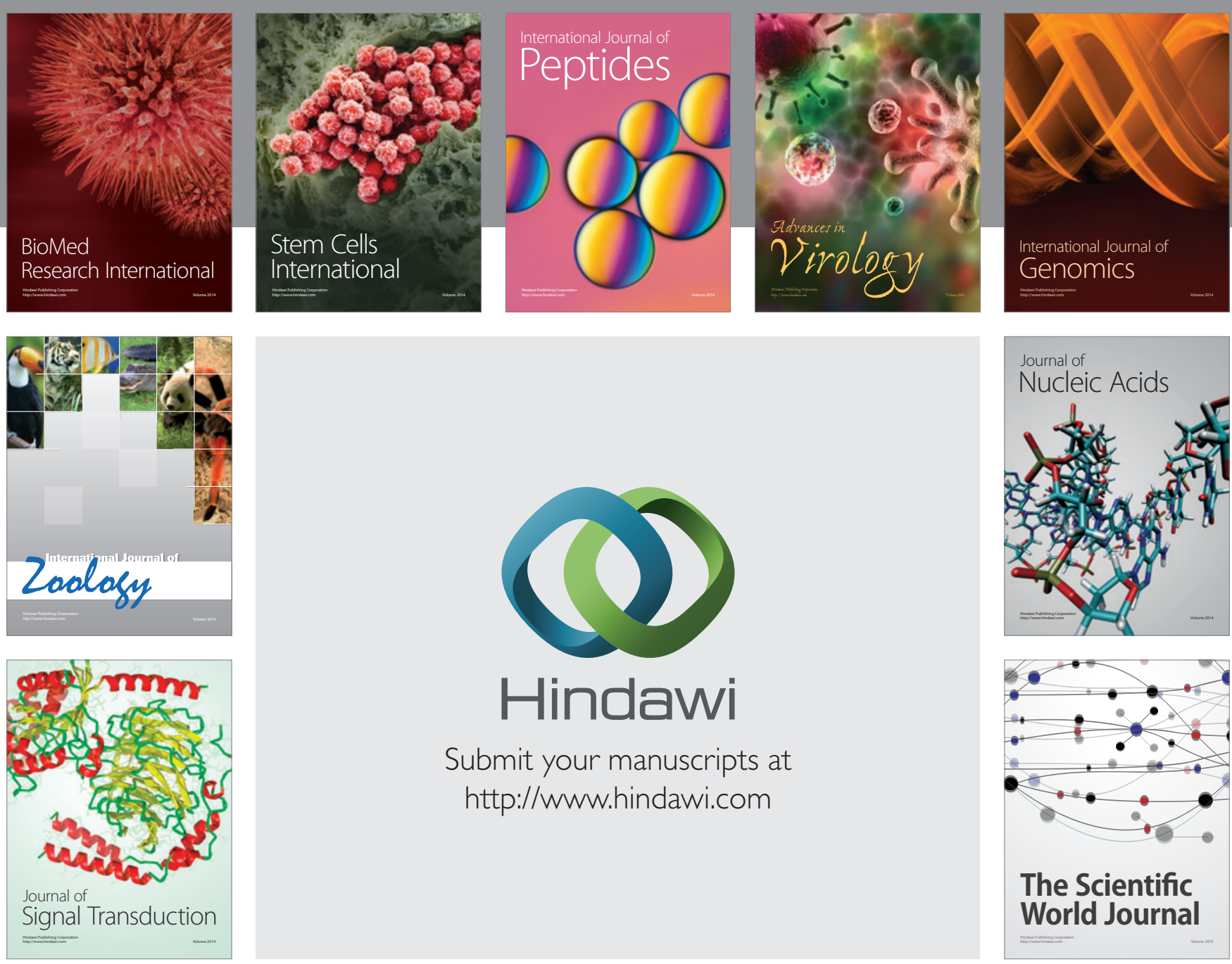

Submit your manuscripts at

http://www.hindawi.com
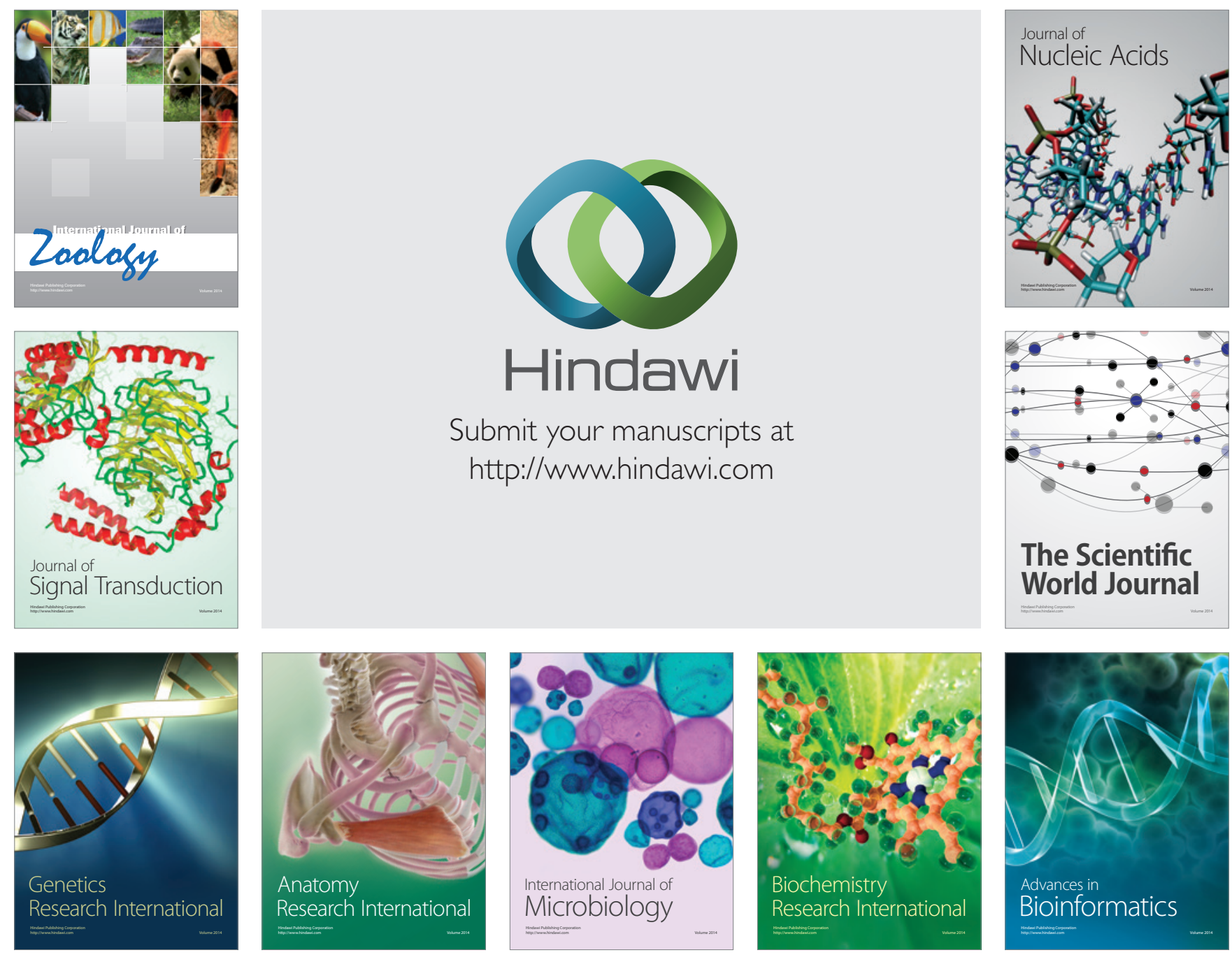

The Scientific World Journal
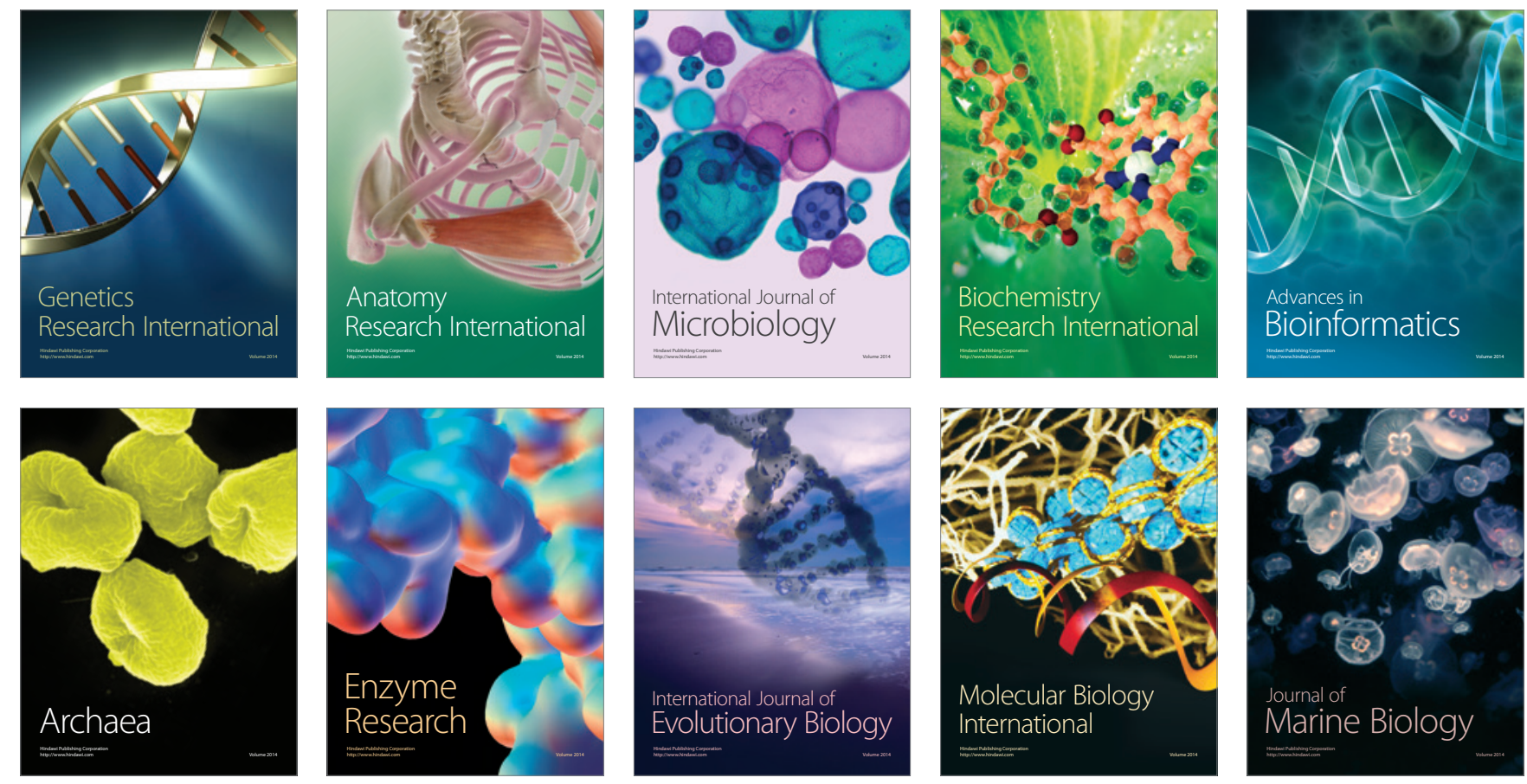